\title{
Retraction: At which magnetic field, exactly, does the Kondo resonance begin to split? \\ A Fermi liquid description of the low-energy properties of the Anderson model [Phys. Rev. B 95, 165404 (2017)]
}

\author{
Michele Filippone, Cătălin Paşcu Moca, Jan von Delft, and Christophe Mora
}

(Q) (Received 10 July 2018; published 6 August 2018)

DOI: 10.1103/PhysRevB.98.079902

The original published version of this paper has been retracted because it contained a trivial but fatal sign error that led to incorrect conclusions. The corrected version, which pinpoints the origin and consequences of the sign error and contains a different set of conclusions, has been published separately as Ref. [1].

[1] M. Filippone, C. P. Moca, A. Weichselbaum, J. von Delft, and C. Mora, Phys. Rev. B 98, 075404 (2018). 